\title{
Anidulafungin Versus Micafungin in the Treatment of Candidemia in Adult Patients
}

\author{
Jin Woong Suh • Sun Bean Kim • Young Kyung Yoon · Jang Wook Sohn • \\ Min Ja Kim • Jong Hun Kim (1)
}

Received: 26 January 2020/ Accepted: 29 June 2020/Published online: 23 July 2020

(C) Springer Nature B.V. 2020

\begin{abstract}
Background Echinocandins are recommended for the treatment of invasive candidiasis and candidemia. However, there are few studies comparing anidulafungin and micafungin in terms of efficacy and safety. The objective of this study was to evaluate the clinical efficacy and safety between anidulafungin and micafungin treatment for adult patients with candidemia. Methods This retrospective cohort study performed on adult candidemia patients diagnosed from January 2006 through December 2018 at a tertiary medical center. The study subjects included adult patients $\geq 19$ years with candidemia who were only treated with anidulafungin or micafungin for $\geq 3$ days. Clinical characteristics were collected and analyzed. Hepatotoxicity was assessed according to the Common Terminology Criteria for Adverse Events Version 5.0.
\end{abstract}

Handling editor: Jean-Pierre Gangneux.

Electronic supplementary material The online version of this article (https://doi.org/10.1007/s11046-020-00471-8) contains supplementary material, which is available to authorized users.

J. W. Suh · S. B. Kim · Y. K. Yoon · J. W. Sohn · M. J. Kim · J. H. Kim $(\bowtie)$

Division of Infectious Diseases, Department of Internal Medicine, Korea University College of Medicine, 73 Inchon-ro, Seongbuk-gu, Seoul 02841, Republic of Korea e-mail: smonti1976@hotmail.com
Results A total of 98 patients with candidemia were treated with anidulafungin $(n=52,53.1 \%)$ or micafungin $(n=46,46.9 \%)$. There were no significant differences in age, sex, source of candidemia, and comorbidities between the anidulafungin and micafungin groups. Although there were more patients with abnormal baseline liver function test (LFT) in the anidulafungin group, the rate of clinical response (51.9\% vs. $46.7 \%)$, mycological response $(76.9 \%$ vs. $67.4 \%$ ), and mortality (30-day mortality $26.9 \%$ vs. $21.7 \%$ and 90 -day mortality $78.8 \%$ vs. $73.9 \%$ ) was similar between the anidulafungin and micafungin groups. Also, there was no significant difference in terms of hepatotoxicity, even among the patients with abnormal baseline LFT between the two groups.

Conclusions Our results suggest that clinical efficacy and safety may be similar between anidulafungin and micafungin treatment for adult patients with candidemia.

Keywords Candidemia - Anidulafungin . Micafungin · Efficacy $\cdot$ Safety

\section{Introduction}

Invasive candidiasis contributes to significant morbidity and mortality in the health care setting [1]. Candidemia is the most frequent manifestation of 
invasive candidiasis, and it represents approximately $10 \%$ of nosocomial infections. Furthermore, it is the fourth most common cause of nosocomial bloodstream infection, requiring prompt diagnosis and treatment to improve outcomes [2]. During the last two decades, the incidence of candidemia has not decreased despite the advancement of diagnostic methods and the implementation of prevention strategies against the acquisition of candidemia. Moreover, the tendency of an increasing proportion of Candida non-albicans such as $C$. tropicalis, $C$. glabrata, and $C$. parapsilosis in candidemia has been noted recently [3-10], raising concerns for the emergence of candidemia with fluconazole resistance. The current Infectious Diseases Society of America (IDSA) guideline recommends using an echinocandin as initial therapy against candidemia based on its favorable efficacy over fluconazole with a low rate of drug resistance [1]. Echinocandins are $N$-acyl-substituted cyclic hexapeptides with potent activity as an inhibitor of $\beta$-(1,3)-glucan synthesis in the fungal cell wall. Currently, there are three echinocandins (anidulafungin, caspofungin, and micafungin) licensed for clinical use $[11,12]$. Pharmacological characteristics are generally similar among echinocandins. Also, echinocandins have a broad spectrum of activity against most Candida species despite the recent emergence of resistance [13-15]. The IDSA guideline states that the clinical efficacy of echinocandins is similar, and echinocandins are considered to be similar in terms of the clinical efficacy based on the limited small number of clinical studies directly comparing different echinocandins [1]. Previous reports from two randomized trials and one retrospective study showed similar results in the efficacy, safety, and costeffectiveness for the treatment of invasive candidiasis between patients treated with micafungin and caspofungin [16-18]. However, there are limited data on comparing anidulafungin and micafungin in the treatment of candidemia in adult patients. Therefore, the current study was designed to evaluate the clinical efficacy and safety between anidulafungin and micafungin treatment for adult patients with candidemia.

\section{Materials and Methods}

Study Design and Patients

This retrospective cohort study was conducted on adult patients admitted to a tertiary care hospital (Korea University Anam Hospital, Seoul, Republic of Korea) from January 2006 to December 2018. Inclusion criteria were (1) adult patients $\geq 19$ years diagnosed with candidemia and (2) receipt of systemic antifungal therapy with anidulafungin or micafungin $\geq 3$ days. Exclusion criteria were (1) patients $<19$ years, (2) patients without candidemia, (3) receipt of systemic antifungal therapy with other agents than anidulafungin or micafungin, (4) receipt of systemic antifungal therapy with anidulafungin or micafungin $<3$ days. Patients' demographics, clinical data including underlying comorbidities, clinical conditions such as the presence of septic shock, receipt of recent surgery, the presence of neutropenia, total parenteral nutrition, central venous catheter, urinary catheter, ventilator, dialysis, use of antibiotics, Candida spp. colonization, clinical course, and in-hospital mortality were collected on a standardized case report form (CRF) after reviewing the electronic medical records. This study was approved by the institutional review board at the Korea University Anam Hospital (IRB Number 2018AN0440). As this was an observational retrospective study, informed consent was not required. Candidemia was defined as at least one positive peripheral blood culture for Candida spp. obtained from an adult hospitalized patient $\geq 19$ years with compatible clinical signs or symptoms [1]. Identification and antifungal susceptibility of Candida spp. from blood culture were performed using the BacT/ALERT $^{\circledR}$ 3D Microbial Detection System (bioMérieux, Inc., Durham, NC, USA) and the automated Vitek $^{\circledR} 2$ Yeast Biochemical Card (bioMérieux, Inc.). The Charlson comorbidity index was calculated to assess the impact of comorbidities. The European Confederation of Medical Mycology Quality of Clinical Candidemia Management score (EQUAL Candida score) [19] was also calculated for the measurement of adherence to the IDSA guideline [1]. Neutropenia was defined as an absolute neutrophil count of $<500$ cells $/ \mathrm{mm}^{3}$. The definition of septic shock was adapted from the third International Consensus Definitions for Sepsis and Septic Shock (Sepsis-3) [20]. The use of immunosuppressive agents was 
defined as follows: use of systemic steroid ( $\geq 20 \mathrm{mg} /$ day of prednisone equivalent), antimetabolites, or use of immunomodulatory agents such as tumor necrosis factor-alpha inhibitors or rituximab. Liver function was assessed before and after anidulafungin or micafungin therapy for the measurement of hepatotoxicity. Abnormal liver function was defined as followings: aspartate aminotransferase (AST) $>45 \mathrm{IU} / \mathrm{L}$, alanine aminotransferase (ALT) $>45 \mathrm{IU} / \mathrm{L}$, total bilirubin $>1.4 \mathrm{mg} / \mathrm{dL}$. The classification of hepatotoxicity was based on the Common Terminology Criteria for Adverse Events (CTCAE) version 5 [21]. Treatment outcomes after the micafungin or anidulafungin therapy were assessed in the followings: (1) clinical response defined as complete or partial clinical response of attributable signs, symptoms, and radiographic findings of candidemia following the systemic antifungal therapy with anidulafungin or micafungin according to the European Organization for the Research and Treatment of Cancer/Mycoses Study Group (EORTC/MSG) criteria [22] (clinical failure was defined as failure to achieve clinical response following the systemic antifungal therapy with anidulafungin or micafungin) (2) mortality defined as 30-day mortality and 90-day mortality, and (3) mycological response defined as eradication of candidemia resulted in negative blood culture following the systemic antifungal therapy with anidulafungin or micafungin (mycological failure was defined as failure to eradicate candidemia following the systemic antifungal therapy with anidulafungin or micafungin).

\section{Statistical Analysis}

SPSS version 23.0 for Windows (SPSS Inc., Chicago, IL, USA) used for the statistical analyses. Categorical variables were analyzed using Pearson's Chi-square test or Fisher's exact test. The Mann-Whitney test was used for continuous variables. Variables with a $P$ value $<0.1$ on comparison analysis were included in a multiple logistic regression analysis to determine risk factors associated with clinical treatment failure. The Kaplan-Meier curves were used for survival analyses between the anidulafungin group and the micafungin group. A $P$ value $<0.05$ was considered to be statistically significant.

\section{Results}

Patients and Clinical Characteristics

During the study period, there were a total of 388 adult patients diagnosed with candidemia. Among them, 278 patients were excluded for lack of antifungal treatment with anidulafungin or micafungin. Of the 110 patients, 12 patients were excluded for receipt of anidulafungin or micafungin $<3$ days. Thus, after application of the inclusion and exclusion criteria, 98 patients who received anidulafungin $(n=52,53.1 \%)$ or micafungin $(n=46,46.9 \%) \geq 3$ days as the effective antifungal therapy against candidemia were included and analyzed in the study. Of the 98 patients, the median age was 72 years, and the median of the Charlson comorbidity index was 3 . The median of the EQUAL Candida score was 15. Malignancy and diabetes mellitus were noted in $46.9 \%$ and $35.7 \%$ of the patients. The majority of the patients had central venous catheter placement $(68.4 \%)$, antibiotic treatment $(89.8 \%)$, and receipt of total parenteral nutrition (96.9\%). However, the rate of Candida spp. colonization was low $(1.0 \%)$. Use of ventilator $(38.8 \%)$, renal replacement therapy $(22.4 \%)$, the presence of septic shock $(37.8 \%)$, neutropenia $(8.2 \%)$, and recent surgery in the current hospital admission prior to candidemia (22.4\%) were noted. The most common source of candidemia was central venous catheterization $(62.2 \%)$. Between the two groups of patients treated with either anidulafungin or micafungin, demographic and baseline clinical characteristics were comparable. However, there were more patients with abnormal baseline AST $(51.0 \%$ vs. $20.5 \%, P=0.002)$ and abnormal baseline total bilirubin (58.3\% vs. $30.2 \%$, $P=0.007$ ) in the anidulafungin group (Table 1).

\section{Fungal Infections}

The most commonly isolated Candida species from candidemia were $C$. albicans $(n=42,42.9 \%), C$. parapsilosis $(n=22,22.4 \%)$, and $C$. tropicalis $(n=20,20.4 \%)$. Other Candida species were $C$. glabrata $(n=7,7.1 \%)$, C. krusei $(n=2,2.0 \%), C$. guilliermondii $(n=3,3.1 \%)$, and $C$. utilis $(n=1$, $1.0 \%$ ). Fluconazole resistance was low in $C$. albicans, C. tropicalis, C. glabrata, C. guilliermondii, and $C$. utilis. However, higher degree of fluconazole resistance was observed in C. parapsilosis $(59.1 \%)$. No 
Table 1 Demographic and baseline clinical characteristics of candidemia patients treated with either anidulafungin or micafungin

\begin{tabular}{|c|c|c|c|c|}
\hline & Total $(n=98)$ & $\begin{array}{l}\text { Anidulafungin } \\
(n=52)\end{array}$ & $\begin{array}{l}\text { Micafungin } \\
(n=46)\end{array}$ & $P$ value \\
\hline Age, median (IQR) years & $72(63-79)$ & $72(65-79)$ & $72(58-79)$ & 0.631 \\
\hline Sex & & & & 0.728 \\
\hline Male, $n(\%)$ & $60(61.2)$ & $31(59.6)$ & $29(63.0)$ & \\
\hline Female, $n(\%)$ & $38(38.8)$ & $21(40.4)$ & $17(37.0)$ & \\
\hline \multicolumn{5}{|l|}{ Comorbid illness } \\
\hline Malignancy, $n(\%)$ & $46(46.9)$ & $24(46.2)$ & $22(47.8)$ & 0.869 \\
\hline Cardiac disease, $n(\%)$ & $41(41.8)$ & $17(32.7)$ & $24(52.2)$ & 0.051 \\
\hline Neurologic disease, $n(\%)$ & $37(37.8)$ & $18(34.6)$ & $19(41.3)$ & 0.495 \\
\hline Renal disease, $n(\%)$ & $36(36.7)$ & $20(38.5)$ & $16(34.8)$ & 0.706 \\
\hline Liver disease, $n(\%)$ & $18(18.4)$ & $11(21.2)$ & $7(15.2)$ & 0.449 \\
\hline Pulmonary disease, $n(\%)$ & $12(12.2)$ & $6(11.5)$ & $6(13.0)$ & 0.821 \\
\hline Diabetes mellitus, $n(\%)$ & $35(35.7)$ & $20(38.5)$ & $15(32.6)$ & 0.546 \\
\hline Charlson comorbidity index, media $n$ (IQR) & $3(1-6)$ & $3(1-6)$ & $3(1-6)$ & 0.832 \\
\hline \multicolumn{5}{|l|}{ Clinical condition } \\
\hline Previous Candida spp. colonization, $n(\%)$ & $1(1.0)$ & $0(0.0)$ & $1(2.2)$ & 0.469 \\
\hline Previous use of antibiotics, $n(\%)$ & $88(89.8)$ & $46(88.5)$ & $42(91.3)$ & 0.746 \\
\hline Central venous catheterization, $n(\%)$ & $67(68.4)$ & $35(67.3)$ & $32(69.6)$ & 0.810 \\
\hline Total parenteral nutrition, $n(\%)$ & $95(96.9)$ & $51(98.1)$ & $44(95.7)$ & 0.487 \\
\hline Use of ventilator, $n(\%)$ & $38(38.8)$ & $20(38.5)$ & $18(39.1)$ & 0.946 \\
\hline Renal replacement therapy, $n(\%)$ & $22(22.4)$ & $13(25.0)$ & $9(19.6)$ & 0.520 \\
\hline Neutropenia, $n(\%)$ & $8(8.2)$ & $4(7.7)$ & $4(8.7)$ & 0.856 \\
\hline Presence of septic shock, $n(\%)$ & $37(37.8)$ & $22(42.3)$ & $15(32.6)$ & 0.323 \\
\hline $\begin{array}{l}\text { Recent surgery in the current admission prior to } \\
\text { candidemia, } n(\%)\end{array}$ & $22(22.4)$ & $14(26.9)$ & $8(17.4)$ & 0.259 \\
\hline \multicolumn{5}{|l|}{ Laboratory test } \\
\hline Baseline AST median (IQR) & $34(23-62)$ & $49(26-70)$ & $28(19-44)$ & 0.017 \\
\hline Abnormal baseline AST $n(\%)$ & $35(35.7)$ & $26(51.0)$ & $9(20.5)$ & 0.002 \\
\hline Baseline $\mathrm{ALT}^{\mathrm{a}}$ median (IQR) & $22(12-44)$ & $27(13-48)$ & $18(11-35)$ & 0.175 \\
\hline Abnormal baseline ALT $n(\%)$ & $18(18.4)$ & $13(25.5)$ & $5(11.4)$ & 0.080 \\
\hline Baseline total bilirubin $^{\mathrm{a}}$ median (IQR) & $1.20(0.74-2.45)$ & $1.61(0.83-3.63)$ & $1.03(0.61-1.51)$ & 0.001 \\
\hline Abnormal baseline total bilirubin, $n(\%)$ & $41(41.8)$ & $28(58.3)$ & $13(30.2)$ & 0.007 \\
\hline Albumin median (IQR) & $2.5(2.3-2.9)$ & $2.5(2.3-2.9)$ & $2.5(2.1-2.8)$ & 0.608 \\
\hline \multicolumn{5}{|l|}{ Source of candidemia, $n(\%)$} \\
\hline Gastrointestinal tract, $n(\%)$ & $12(12.2)$ & $5(9.6)$ & $7(15.2)$ & 0.399 \\
\hline Central venous catheter, $n(\%)$ & $61(62.2)$ & $32(61.5)$ & $29(63.0)$ & 0.878 \\
\hline Genitourinary tract, $n(\%)$ & $7(7.1)$ & $5(9.6)$ & $2(4.3)$ & 0.312 \\
\hline Abscess, $n(\%)$ & $2(2.0)$ & $1(1.9)$ & $1(2.2)$ & 0.930 \\
\hline Others or unknown, $n(\%)$ & $16(16.3)$ & $9(17.3)$ & $7(15.2)$ & 0.780 \\
\hline \multicolumn{5}{|l|}{ Antifungal treatment } \\
\hline Duration of treatment (days), median (IQR) & $14(7-18)$ & $14(5-17)$ & $15(10-19)$ & 0.114 \\
\hline EQUAL Candida score ${ }^{\mathrm{b}}$, median (IQR) & $15(15-15)$ & $15(15-15)$ & $15(14-16)$ & 0.871 \\
\hline \multicolumn{5}{|l|}{ Treatment outcomes } \\
\hline Duration of treatment (days), median (IQR) & $14(7-18)$ & $14(5-17)$ & $15(10-19)$ & 0.114 \\
\hline Clinical response, $n(\%)$ & $48(49.5)$ & $27(51.9)$ & $21(46.7)$ & 0.606 \\
\hline
\end{tabular}


Table 1 continued

\begin{tabular}{|c|c|c|c|c|}
\hline & Total $(n=98)$ & $\begin{array}{l}\text { Anidulafungin } \\
(n=52)\end{array}$ & $\begin{array}{l}\text { Micafungin } \\
(n=46)\end{array}$ & $P$ value \\
\hline Mycological response, $n(\%)$ & $71(72.4)$ & $40(76.9)$ & $31(67.4)$ & 0.292 \\
\hline Mortality day 30 after treatment, $n(\%)$ & $24(24.5)$ & $14(26.9)$ & $10(21.7)$ & 0.551 \\
\hline Mortality day 90 after treatment, $n(\%)$ & $75(76.5)$ & $41(78.8)$ & $34(73.9)$ & 0.565 \\
\hline
\end{tabular}

$I Q R$ interquartile range, $A S T$ aspartate aminotransferase IU/L, data available for 44 cases of micafungin group and 51 cases of anidulafungin group, $A L T$ alanine aminotransferase IU/L, data available 44 cases of micafungin group and 51 cases of anidulafungin group

${ }^{\mathrm{a}}$ Total bilirubin $\mathrm{mg} / \mathrm{dL}$, data available for 43 cases of micafungin group and 48 cases of anidulafungin group

${ }^{\mathrm{b}}$ EQUAL Candida score, The European Confederation of Medical Mycology Quality of Clinical Candidemia Management score

echinocandin resistance was noted in Candida species. There was no difference in the distribution of Candida species between the anidulafungin and micafungin groups (Table 2).

\section{Outcomes}

The median duration of treatment was 14 days for the anidulafungin group and 15 days for the micafungin group without a statistical difference, $P=0.114$. There was no difference in terms of the EQUAL Candida score between the anidulafungin and micafungin group (median 15 vs. $15, P=0.871$ ). The mycological response was not significantly different between the anidulafungin (76.9\%) group and micafungin $(67.4 \%)$ group, $P=0.292$. Also, there was no significant difference in the clinical response between the anidulafungin $(51.9 \%)$ group and micafungin

Table 2 Candida species identified from candidemia patients treated with either anidulafungin or micafungin

\begin{tabular}{|c|c|c|c|c|}
\hline Candida species, $n(\%)$ & Total $(n=98)$ & Anidulafugin $(n=52)$ & Micafungin $(n=46)$ & $P$ value \\
\hline C. albicans, $n(\%)$ & $42(42.9)$ & $23(44.2)$ & $19(41.3)$ & 0.770 \\
\hline Fluconazole susceptibility (\%) & $40 / 42(95.2)$ & $23 / 23(100.0)$ & $17 / 19(89.5)$ & 0.199 \\
\hline Caspofungin susceptibility (\%) & $41 / 41(100.0)$ & $22 / 22(100.0)$ & $19 / 19(100.0)$ & NA \\
\hline C. parapsilosis, $n(\%)$ & $22(22.4)$ & $9(17.3)$ & $13(28.3)$ & 0.195 \\
\hline Fluconazole susceptibility (\%) & $13 / 22(59.1)$ & $4 / 9(44.4)$ & $9 / 13(69.2)$ & 0.384 \\
\hline Caspofungin susceptibility (\%) & $21 / 21(100.0)$ & $8 / 8(100.0)$ & $13 / 13(100.0)$ & NA \\
\hline C. tropicalis, $n(\%)$ & $20(20.4)$ & $12(23.1)$ & $8(17.4)$ & 0.486 \\
\hline Fluconazole susceptibility (\%) & $20 / 20(100.0)$ & $12 / 12(100.0)$ & $8 / 8(100.0)$ & NA \\
\hline Caspofungin susceptibility (\%) & $20 / 20(100.0)$ & $12 / 12(100.0)$ & $8 / 8(100.0)$ & NA \\
\hline C. glabrata, $n(\%)$ & $7(7.1)$ & $5(9.6)$ & $2(4.3)$ & 0.312 \\
\hline Fluconazole susceptibility (\%) & 6/6 (100.0) & 4/4 (100.0) & $2 / 2(100.0)$ & NA \\
\hline Caspofungin susceptibility (\%) & $6 / 6(100.0)$ & $4 / 4(100.0)$ & $2 / 2(100.0)$ & NA \\
\hline C. krusei, $n(\%)$ & $2(2.0)$ & $1(1.9)$ & $1(2.2)$ & $0.930^{\prime}$ \\
\hline Fluconazole susceptibility (\%) & $0 / 2(0.0)$ & $0 / 1(0.0)$ & $0 / 1(0.0)$ & NA \\
\hline Caspofungin susceptibility (\%) & $2 / 2(100.0)$ & $1 / 1(100.0)$ & $1 / 1(100.0)$ & NA \\
\hline Others, $n(\%)$ & $5(5.1)$ & $2^{\mathrm{b}}(3.8)$ & $3^{a}(6.5)$ & 0.548 \\
\hline Fluconazole susceptibility (\%) & $5 / 5(100.0)$ & $2 / 2(100.0)$ & $3 / 3(100.0)$ & NA \\
\hline Caspofungin susceptibility (\%) & $5 / 5(100.0)$ & $2 / 2(100.0)$ & $3 / 3(100.0)$ & NA \\
\hline
\end{tabular}

$N A$ not available

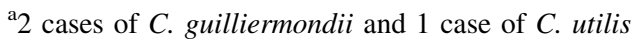

$\mathrm{b}_{2}$ cases of $C$. guilliermondii 
(46.7\%) group, $P=0.606$ (Table 1). To identify factors associated with clinical response and failure, comparison analysis was performed. There was no difference in the types of antifungal therapy [anidulafungin $(50.0 \%)$ vs. micafungin $(50.0 \%), P=0.535]$ in the clinical failure group. However, there were more patients with abnormal baseline total bilirubin (57.4\% vs. $31.8 \%, P=0.014)$, renal replacement therapy (36.0\% vs. $8.3 \%, P=0.001)$, septic shock $(62.0 \%$ vs. $12.5 \%, P<0.001)$, EQUAL Candida score $<15$ (32.0\% vs. $8.3 \%, P=0.004)$, and mycological failure (50.0\% vs. $4.2 \%, P<0.001)$ in the clinical failure group than in the clinical response group (Table 3 ). In the multivariate logistic regression analysis, septic shock [odds ratio (OR) 5.373, 95\% confidence interval (CI) $1.580-18.270, P=0.007]$, renal replacement therapy (OR 4.512, 95\% CI 1.061-19.184, $P=0.041)$, and failure of mycological response (OR 14.905, 95\% CI 2.905-76.467, $P=0.001)$ were significantly associated with clinical failure. There was no difference in the 30 -day mortality $(26.9 \%$ vs. $21.7 \%, P=0.551)$ and 90 -day mortality $(78.8 \%$ vs. $73.9 \%, P=0.565)$ between the anidulafungin group and micafungin group (Table 1). The Kaplan-Meier curves with the log-rank test indicated that there were no significant differences in survival of 30-day $(P=0.590)$ and 90 -day $(P=0.425)$ between the anidulafungin group and micafungin group (Figs. 1, 2). However, there were more patients with EQUAL Candida score $<15$ in the 30-day mortality group (45.8\% vs. $12.2 \%, P=0.001$ ) than in the 30 -day survivor group. Moreover, the Kaplan-Meier curves with the log-rank test showed that there was a significant difference in survival of 30-day $(P<0.001)$ between patients with EQUAL Candida score $<15$ and patients with EQUAL Candida score $\geq 15$ (Supplementary Figure 1). On the contrary, there was no difference in the proportion of patients with EQUAL Candida score $<15$ between 90-day mortality group and 90 -day survivor group $(22.7 \%$ vs. $13.0 \%, P=0.389$ ).

\section{Adverse Events}

The treatment-emergent adverse events of hepatotoxicity defined as CTCAE $\geq 1$ were similar between the anidulafungin (57.7\%) group and micafungin (47.8\%) group, $P=0.418$. Also, there was no significant difference in the occurrence of severe hepatotoxicity defined as CTCAE $\geq 3$ between the anidulafungin $(11.5 \%)$ group and micafungin $(13.0 \%)$ group, $P=0.821$. These patterns of similar distribution of adverse events of hepatotoxicity were consistently observed in the patients with abnormal baseline liver function test (LFT) as well: CTCAE $\geq 1$ (anidulafungin group $56.4 \%$ vs. micafungin group $61.9 \%$, $P=0.786$ ) and CTCAE $\geq 3$ (anidulafungin group $10.3 \%$ vs. micafungin group $14.3 \%, P=0.687$ ). Nephrotoxicity was noted in one patient in the micafungin group and none in the anidulafungin group. There were no other noticeable adverse events such as skin rash and vomiting.

\section{Discussion}

In the current study, we compared the efficacy and safety of different echinocandins (anidulafungin vs. micafungin) for the treatment of candidemia among hospitalized adult patients, including critically ill intensive care unit patients. Both the clinical and mycological response were similar in the anidulafungin group and micafungin group. Also, the mortality rate did not differ between the two groups. Therefore, our results suggest that there are no significant differences in terms of clinical efficacy and safety for the treatment of candidemia in adult patients with either anidulafungin or micafungin. Our results are in line with the previous studies, which reported similar efficacy and safety between different echinocandins for the treatment of invasive candidiasis, including candidemia (micafungin vs. caspofungin [18], micafungin vs. anidulafungin [23]). However, one of the distinct features of our study is that we included and evaluated hospitalized adult candidemia patients after excluding invasive candidiasis patients without candidemia. Thus, our study results could be useful for considerations of antifungal treatment for candidemia, which is the most common form of invasive candidiasis in the hospital setting.

The recommended duration of antifungal treatment for candidemia is 14 days after the first negative blood culture [1]. The patients in the anidulafungin group and micafungin group received a median duration of 14 days and 15 days, respectively. This is in line with the guideline recommendation [1], and the clinical response rate was similar between the micafungin group and anidulafungin group. However, the clinical 
Table 3 Characteristics of candidemia patients stratified to clinical response and clinical failure following treatment with either anidulafungin or micafungin

\begin{tabular}{|c|c|c|c|}
\hline & $\begin{array}{l}\text { Clinical response } \\
(n=48)\end{array}$ & $\begin{array}{l}\text { Clinical failure } \\
(n=50)\end{array}$ & $P$ value \\
\hline Age $\geq 70, n(\%)$ & $27(56.3)$ & $31(62.0)$ & 0.563 \\
\hline Sex & & & 0.872 \\
\hline Male, $n(\%)$ & $29(60.4)$ & $31(62.0)$ & \\
\hline Female, $n(\%)$ & $19(39.6)$ & $19(38.0)$ & \\
\hline \multicolumn{4}{|l|}{ Comorbid illness } \\
\hline Malignancy, $n(\%)$ & $20(41.7)$ & $26(52.0)$ & 0.306 \\
\hline Cardiac disease, $n(\%)$ & $21(43.8)$ & $20(40.0)$ & 0.707 \\
\hline Neurologic disease, $n(\%)$ & $17(35.4)$ & $20(40.0)$ & 0.640 \\
\hline Renal disease, $n(\%)$ & $14(29.2)$ & $22(44.0)$ & 0.128 \\
\hline Liver disease, $n(\%)$ & $10(20.8)$ & $8(16.0)$ & 0.537 \\
\hline Pulmonary disease, $n(\%)$ & $5(10.4)$ & $7(14.0)$ & 0.589 \\
\hline Diabetes mellitus, $n(\%)$ & $18(37.5)$ & $17(34.0)$ & 0.718 \\
\hline \multicolumn{4}{|l|}{ Clinical condition } \\
\hline Previous Candida spp. Colonization, $n(\%)$ & $1(2.1)$ & $0(0.0)$ & 0.490 \\
\hline Previous use of antibiotics, $n(\%)$ & $44(91.7)$ & $44(88.0)$ & 0.741 \\
\hline Central venous catheterization, $n(\%)$ & $30(62.5)$ & $37(74.0)$ & 0.221 \\
\hline Total parenteral nutrition, $n(\%)$ & $47(97.9)$ & $48(96.0)$ & 1.000 \\
\hline Use of ventilator, $n(\%)$ & $14(29.2)$ & $24(48.0)$ & 0.056 \\
\hline Renal replacement therapy, $n(\%)$ & $4(8.3)$ & $18(36.0)$ & 0.001 \\
\hline Neutropenia, $n(\%)$ & $1(2.1)$ & $7(14.0)$ & 0.060 \\
\hline Presence of septic shock, $n(\%)$ & $6(12.5)$ & $31(62.0)$ & $<0.001$ \\
\hline Recent surgery in the current admission prior to candidemia, $n(\%)$ & $11(22.9)$ & $11(22.0)$ & 0.913 \\
\hline \multicolumn{4}{|l|}{ Laboratory test } \\
\hline Abnormal baseline AST, $n(\%)$ & $21(43.8)$ & $14(29.8)$ & 0.158 \\
\hline Abnormal baseline ALT, $n(\%)$ & $13(27.1)$ & $5(10.6)$ & 0.041 \\
\hline Abnormal baseline total bilirubin ${ }^{\mathrm{a}}, n(\%)$ & $14(31.8)$ & $27(57.4)$ & 0.014 \\
\hline \multicolumn{4}{|l|}{ Source of candidemia } \\
\hline Gastrointestinal tract, $n(\%)$ & $5(10.4)$ & $7(14.0)$ & 0.589 \\
\hline Central venous catheter, $n(\%)$ & $28(58.3)$ & $33(66.0)$ & 0.434 \\
\hline Genitourinary tract, $n(\%)$ & $4(8.3)$ & $3(6.0)$ & 0.712 \\
\hline Abscess, $n(\%)$ & $1(2.1)$ & $1(2.0)$ & 1.000 \\
\hline Others or unknown, $n(\%)$ & $10(20.8)$ & $6(12.0)$ & 0.237 \\
\hline \multicolumn{4}{|l|}{ Antifungal treatment } \\
\hline Anidulafungin, $n(\%)$ & $27(56.3)$ & $25(50.0)$ & 0.535 \\
\hline Micafungin, $n(\%)$ & $21(43.8)$ & $25(50.0)$ & \\
\hline EQUAL Candida score $^{\mathrm{b}} \geq 15, n(\%)$ & $44(91.7)$ & $34(68.0)$ & 0.004 \\
\hline$<15, n(\%)$ & $4(8.3)$ & $16(32.0)$ & \\
\hline Mycological response, $n(\%)$ & $46(95.8)$ & $25(50.0)$ & $<0.001$ \\
\hline Mycological failure, $n(\%)$ & $2(4.2)$ & $25(50.0)$ & \\
\hline
\end{tabular}

$A S T$ aspartate aminotransferase IU/L

$A L T$ alanine aminotransferase IU/L

${ }^{\mathrm{a}}$ Total bilirubin $\mathrm{mg} / \mathrm{dL}$

${ }^{\mathrm{b}}$ EQUAL Candida score, The European Confederation of Medical Mycology Quality of Clinical Candidemia Management score 


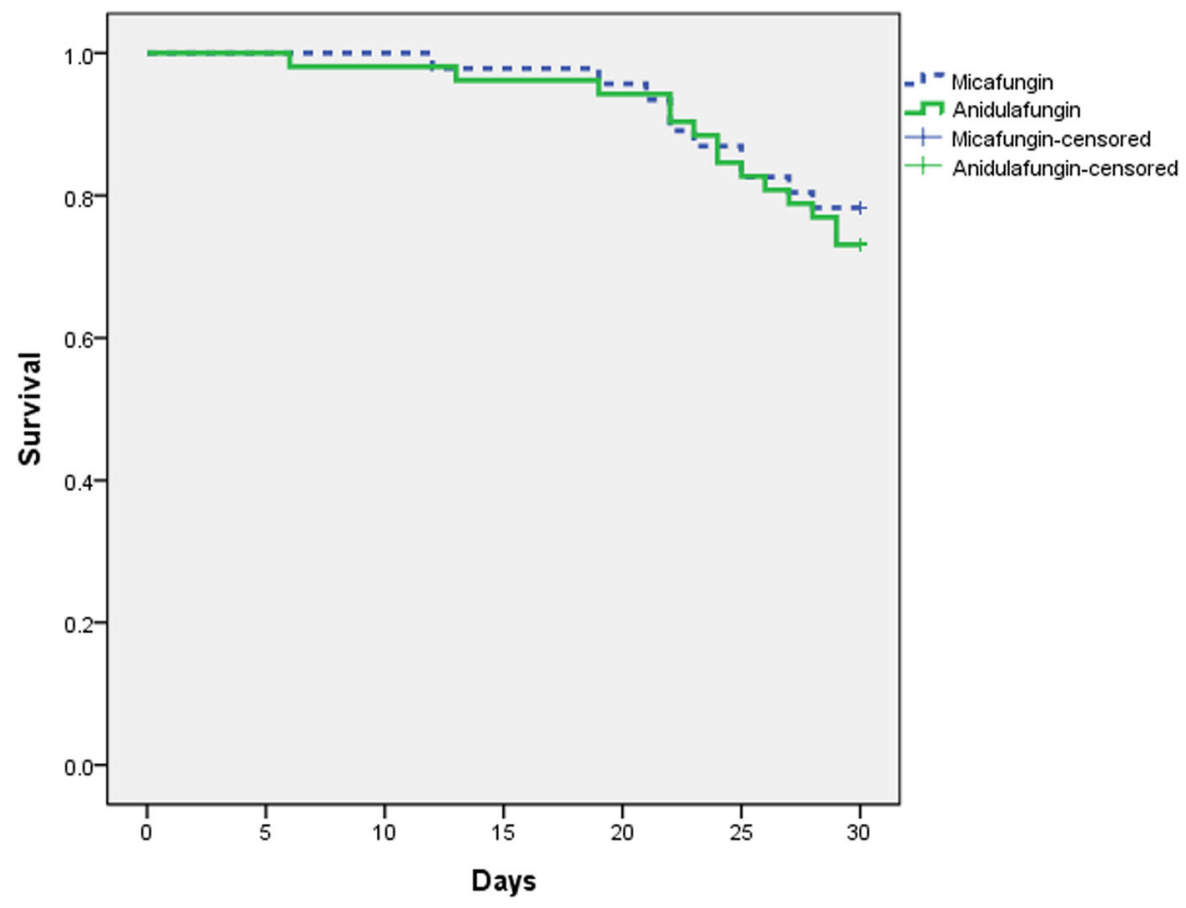

Fig. 1 Kaplan-Meier survival curve after initiation of antifungal therapy with anidulafungin or micafungin. Survival of 30 day, $P=0.590$ (log-rank test) Anidulafungin survival rate $73.1 \%$ versus micafungin survival rate $78.3 \%$

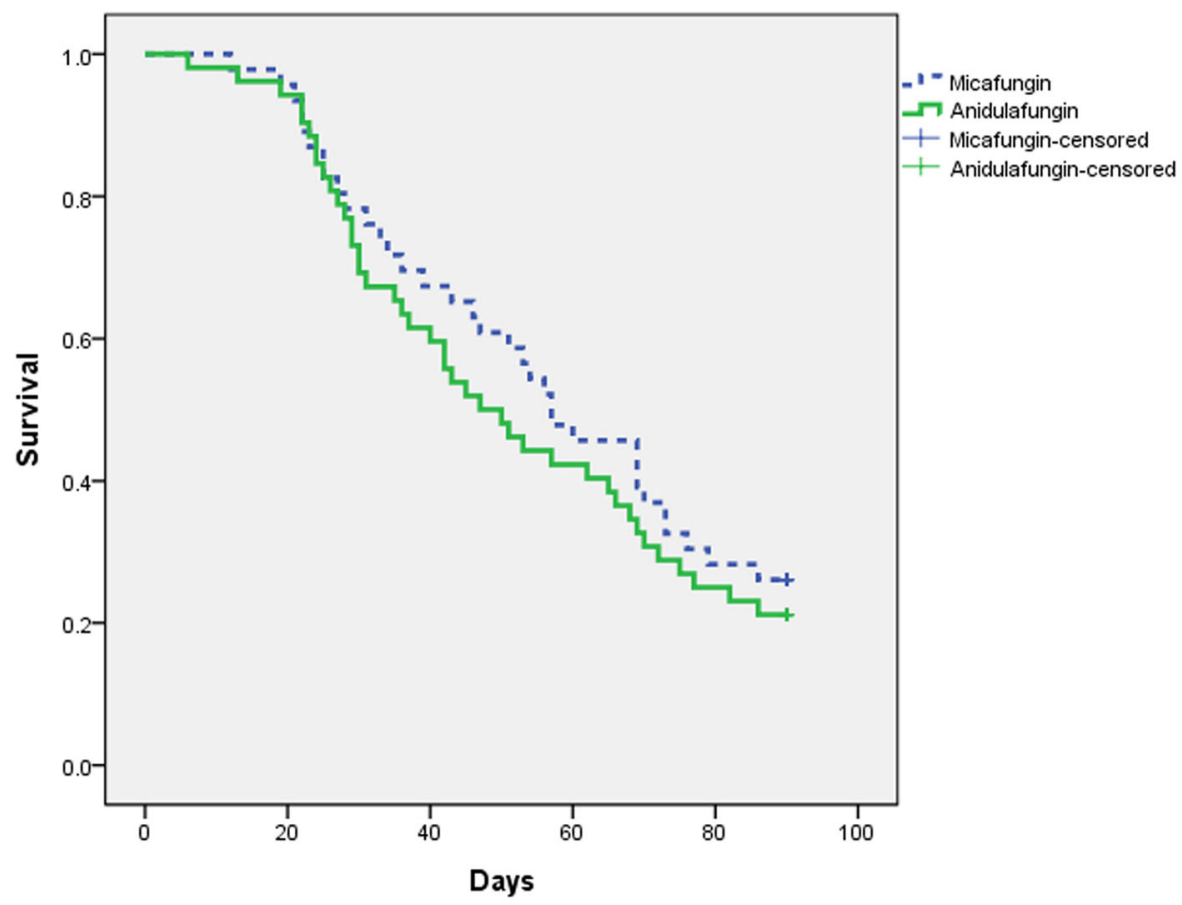

Fig. 2 Kaplan-Meier survival curve after initiation of antifungal therapy with anidulafungin or micafungin. Survival of 90 day, $P=$ 0.425 (log-rank test) Anidulafungin survival rate $21.2 \%$ versus micafungin survival rate $26.1 \%$ 
response rate from our study was lower than those of previous studies [18, 23]. There are several factors for consideration. First, our study patients were older with a median age of 72 years. Decreased immunological response to infection by inadequate phagocytosis, diminished function and number of T-cells and B-cells, and altered cytokine production have been shown to be in relation to the aging $[24,25]$. Second, the proportion of patients with shock was higher in this study than in the previous study [24]. Moreover, while our study included all candidemia patients, previous studies included both candidemia and non-candidemia patients diagnosed with invasive candidiasis. As approximately $30 \%$ of candidemia patients might develop septic shock [26], the higher prevalence of candidemia in our study may suggest an additional indirect risk factor for developing septic shock. Taken together, these factors might have contributed to a lower clinical response rate in our study. In addition, our results of a significant association between failure of mycological response and failure of clinical response were in agreement with a previous study [27], which reported that persistent candidemia is associated with increased adverse outcomes. Thus, these results reaffirm the importance of prompt initiation of effective antifungal treatment against candidemia for optimal clinical response. As there was no significant difference in the mycological response between the anidulafungin group and micafungin group, our results suggest the mycological response rate of anidulafungin is comparable to that of micafungin. Furthermore, the rate of mycological response was similar to a previous study [23]. In line with these findings, our data indicate that echinocandins such as anidulafungin or micafungin are highly effective in the eradication of candidemia, even in the various clinical settings including candidemia patients with critical ill conditions. Interestingly, the rate of mycological response was higher than that of the clinical response in the current study. This result suggests that the clinical response rate might reflect the multifactorial process affected by various clinical factors such as age, comorbidities, and critical illness as well as mycological response factor. Thus, these clinical factors might have influenced the clinical response rate in our study. There were no discernible differences in terms of mortality 30 and 90 days after anidulafungin or micafungin treatment, which is consistent with a previous study comparing different echinocandins [23]. Taken together with the similar clinical response rate and mycological response rate, our results demonstrate that anidulafungin or micafungin can be considered interchangeable for the treatment of candidemia, as recommended in the guideline [1].

The rate of fluconazole resistance against $C$. parapsilosis candidemia in our study was higher $(59.1 \%)$ than those of previously reported studies (2-6\%) [28-30]. Recently, the emergence of fluconazole resistance of $C$. parapsilosis from intensive care units $(30-75 \%)$ has been reported [31-33]. The possible mechanism of fluconazole resistance may include genetic mutation (e.g., mutation of the ERG11 gene) $[34,35]$. Also, certain clinical factors such as previous exposure of antifungal agents, ventilation, and transfusion have been associated with fluconazole resistance of $C$. parapsilosis candidemia [31]. Moreover, a widespread mutation of the ERG11 gene was reported in fluconazole-resistant $C$. parapsilosis bloodstream isolates from a multicenter study in the Republic of Korea [35]. Therefore, our result of a high rate of fluconazole resistance of $C$. parapsilosis might have been due to the possibility of clonal transmission of the fluconazole-resistant mutation gene [35] or multifactorial process affected by various clinical risk factors.

Our results of the low incidence of adverse events other than hepatotoxicity are in agreement of a previous study that echinocandins are known to have favorable safety profiles [1]. However, transient elevation of LFT can occur in up to $15 \%$ of patients treated with an echinocandin [36]. Micafungin is metabolized in the liver by $N$-acetylation, while anidulafungin undergoes spontaneous degradation [37]. Due to this difference in the metabolism, anidulafungin is expected to have a lower hepatotoxicity risk and is often selected in patients with elevated LFT, as seen in our study. However, a recent prospective observational study [38] showed that the majority of patients with preexisting liver injury tolerated micafungin well, supporting the safety of micafungin in patients with the preexisting liver injury with FDA (US Food and Drug Administration) recommendation of no dosage adjustment requirement in patients with hepatic impairment [39]. Our results of a similar rate of hepatotoxicity of patients, including abnormal baseline LFT, between the anidulafungin group and micafungin group are in line with 
previous studies [23, 40]. Moreover, these results suggest that there may be no significant difference in terms of hepatotoxicity from the use of anidulafungin or micafungin for the treatment of candidemia. However, the incidence of hepatotoxicity of our study was higher than those reported in the previous studies $[23,36]$. Abnormal LFT can occur up to $60 \%$ of critically ill patients by shock, ventilation, and renal replacement therapy [41]. Furthermore, more than one-third of our cohort patients were found to have these clinical factors. Thus, we believe that a higher incidence of hepatotoxicity in our study might reflect augmented multifactorial hepatotoxicity effects from these clinical factors, rather than specific events caused by echinocandins themselves.

Although there was no significant difference in the EQUAL Candida score between the two groups of patients treated by anidulafungin and micafungin, we found that lower EQUAL Candida score was noted in the patients with clinical failure as well as in the patients with 30-day mortality. These results are in line with a previous study [42], which reported that greater adherence to the guideline with a higher EQUAL Candida score was associated with survival among candidemia patients. Therefore, our results suggest the potential utility of the EQUAL Candida score as one of the predictors of mortality in candidemia patients. Further research studies may be required to assess the validation of the EQUAL Candida score and to determine the optimal cutoff score point for the ideal survival prediction model.

This study has some limitations, mainly due to a single-center study with a relatively small sample size and retrospective study design. Therefore, unintended selection bias and confounding effects from unmeasured variables might have affected our analyses. Of note, there were no cases of candidemia with echinocandin resistance in our study while the proportion of non- $C$. albicans candidemia was approximately $60 \%$ including $7 \%$ of C. glabrata. Echinocandin resistance has been emerging recently, most commonly in C. glabrata isolates [43]. Thus, our results might not be generalizable to the clinical setting, where there is a higher rate of echinocandin resistance. Furthermore, we did not examine genetic mutation and clinical risk factors for the resistance of antifungal agents, including fluconazole resistance against $C$. parapsilosis, to test our hypothesis of their possible contribution to the emergence of antifungal resistance. In addition, we were unable to perform an adjusted analysis of hepatotoxicity with respect to clinical factors, which might have contributed to a higher incidence of hepatotoxicity in our study. Also, our results of discordant clinical and mycological response might have been affected by the timing and period of the evaluation [23]. However, we used consistent definitions for data collection through the careful review of the medical records to minimize the potential bias. Also,

\section{Conclusion}

In conclusion, our study results suggest that clinical efficacy and safety may be similar between anidulafungin and micafungin treatment for adult candidemia patients, including critically ill patients.

Author Contributions JWS, JHK, and MJK were involved in the conception and the design of the study project. All authors contributed to the collection of study data. JWS and JHK were involved in the analyses of study data. JWS drafted the manuscript and JHK revised the manuscript.

Funding The authors received no financial support for the research, authorship, and publication of this article.

\section{Compliance with Ethical Standards}

Conflict of interest The authors declare that they have no conflict of interest.

\section{References}

1. Pappas PG, Kauffman CA, Andes DR, et al. Clinical practice guideline for the management of Candidiasis: 2016 Update by the Infectious Diseases Society of America. Clin Infect Dis. 2016;62:e1-50.

2. Ghrenassia E, Mokart D, Mayaux J, et al. Candidemia in critically ill immunocompromised patients: report of a retrospective multicenter cohort study. Ann Intensive Care. 2019;9:62.

3. Mencarini J, Mantengoli E, Tofani L, et al. Evaluation of candidemia and antifungal consumption in a large tertiary care Italian hospital over a 12-year period. Infection. 2018;46:469-76.

4. Astvad KMT, Johansen HK, Røder BL, et al. Update from a 12-Year nationwide fungemia surveillance: increasing intrinsic and acquired resistance causes concern. J Clin Microbiol. 2018;56(4):e01564-17. 
5. Chapman B, Slavin M, Marriott D, et al. Changing epidemiology of candidaemia in Australia. $\mathbf{J}$ Antimicrob Chemother. 2017;72:1103-8.

6. Diekema D, Arbefeville S, Boyken L, Kroeger J, Pfaller M. The changing epidemiology of healthcare-associated candidemia over three decades. Diagn Microbiol Infect Dis. 2012;73:45-8.

7. Guinea J. Global trends in the distribution of Candida species causing candidemia. Clin Microbiol Infect. 2014;20:5-10.

8. Wisplinghoff $\mathrm{H}$, Ebbers J, Geurtz L, et al. Nosocomial bloodstream infections due to Candida spp. in the USA: species distribution, clinical features and antifungal susceptibilities. Int J Antimicrob Agents. 2014;43:78-81.

9. Ishikane M, Hayakawa K, Kutsuna S, Takeshita N, Ohmagari N. Epidemiology of blood stream infection due to Candida Species in a Tertiary Care Hospital in Japan over 12 years: importance of peripheral line-associated candidemia. PLoS ONE. 2016;11:e0165346.

10. Pfaller MA, Diekema DJ. Epidemiology of invasive candidiasis: a persistent public health problem. Clin Microbiol Rev. 2007;20:133-63.

11. Walsh TJ, Azie N, Andes DR. Development of new strategies for echinocandins: progress in translational research. Clin Infect Dis. 2015;61:S601-3.

12. Denning DW. Echinocandin antifungal drugs. Lancet. 2003;362:1142-51.

13. Kofla G, Ruhnke M. Pharmacology and metabolism of andiulafungin, caspofungin and micafungin in the treatment of invasive candidosis: review of the literature. Eur J Med Res. 2011;16:159-66.

14. Wasmann RE, Muilwijk EW, Burger DM, Verweij PE, Knibbe CA, Brüggemann RJ. Clinical pharmacokinetics and pharmacodynamics of micafungin. Clin Pharmacokinet. 2018;57:267-86.

15. Cornely OA, Marty FM, Stucker F, Pappas PG, Ullmann AJ. Efficacy and safety of micafungin for treatment of serious Candida infections in patients with or without malignant disease. Mycoses. 2011;54:e838-47.

16. Neoh CF, Liew D, Slavin MA, et al. Economic evaluation of micafungin versus caspofungin for the treatment of candidaemia and invasive candidiasis. Intern Med J. 2013;43: 668-77.

17. Kohno S, Izumikawa K, Yoshida M, et al. A double-blind comparative study of the safety and efficacy of caspofungin versus micafungin in the treatment of candidiasis and aspergillosis. Eur J Clin Microbiol Infect Dis. 2013;32: 387-97.

18. Pappas PG, Rotstein CM, Betts RF, et al. Micafungin versus caspofungin for treatment of candidemia and other forms of invasive candidiasis. Clin Infect Dis. 2007;45:883-93.

19. Mellinghoff SC, Hoenigl M, Koehler P, Kumar A, Lagrou $\mathrm{K}$, Lass-Flörl C, et al. EQUAL Candida Score: an ECMM score derived from current guidelines to measure QUAlity of Clinical Candidaemia Management. Mycoses. 2018; 61(5):326-30.

20. Singer M, Deutschman CS, Seymour CW, et al. The third international consensus definitions for sepsis and septic shock (Sepsis-3). JAMA. 2016;315:801-10.

21. https://ctep.cancer.gov/protocoldevelopment/electronic applications/docs/CTCAE_v5_Quick_Reference_8.5x11. pdf. Accessed on 10 April 2019.
22. Segal BH, Herbrecht R, Stevens DA, et al. Defining responses to therapy and study outcomes in clinical trials of invasive fungal diseases: Mycoses Study Group and European Organization for Research and Treatment of Cancer consensus criteria. Clin Infect Dis. 2008;47:674-83.

23. van der Geest PJ, Hunfeld NGM, Ladage SE, Groeneveld ABJ. Micafungin versus anidulafungin in critically ill patients with invasive candidiasis: a retrospective study. BMC Infect Dis. 2016;16:490.

24. Hof H, Mikus G. Candida infections in the elderly. Z Gerontol Geriatr. 2013;46:64-70.

25. Aw D, Silva AB, Palmer DB. Is thymocyte development functional in the aged? Aging (Albany NY). 2009;1: 146-53.

26. Delaloye J, Calandra T. Invasive candidiasis as a cause of sepsis in the critically ill patient. Virulence. 2014;5:161-9.

27. Morrell M, Fraser VJ, Kollef MH. Delaying the empiric treatment of candida bloodstream infection until positive blood culture results are obtained: a potential risk factor for hospital mortality. Antimicrob Agents Chemother. 2005; 49:3640-5.

28. Cleveland AA, Farley MM, Harrison LH, Stein B, Hollick R, Lockhart SR, Magill SS, Derado G, Park BJ. Multilingualism for access, language development and language intellectualisation. Clin Infect Dis. 2012;55(10):1352-61.

29. Berkow EL, Lockhart SR. Fluconazole resistance in Candida species: a current perspective. Infect Drug Resist. 2017;10: 237-45.

30. Pote ST, Sonawane MS, Rahi P, Shah SR, Shouche YS, Patole MS, et al. Distribution of pathogenic yeasts in different clinical samples: their identification, antifungal susceptibility pattern, and cell invasion assays. Infect Drug Resist. 2020;13:1133-45.

31. Aldardeer NF, Albar H, Al-Attas M, Eldali A, Qutub M, Hassanien A, et al. Antifungal resistance in patients with Candidaemia: a retrospective cohort study. BMC Infect Dis. 2020;20(1):1-7.

32. Pinhati HMS, Casulari LA, Souza ACR, Siqueira RA, Damasceno CMG, Colombo AL. Outbreak of candidemia caused by fluconazole resistant Candida parapsilosis strains in an intensive care unit. BMC Infect Dis. 2016;16(1):4-9.

33. Thomaz DY, De Almeida JN, Lima GME, De Oliveira Nunes M, Camargo CH, De Carvalho Grenfell R, et al. An azole-resistant Candida parapsilosis outbreak: clonal persistence in the intensive care unit of a Brazilian teaching hospital. Front Microbiol. 2018;9:2997.

34. Souza ACR, Fuchs BB, Pinhati HMS, Siqueira RA, Hagen $\mathrm{F}$, Meis JF, et al. Candida parapsilosis resistance to fluconazole: Molecular mechanisms and in vivo impact in infected Galleria mellonella larvae. Antimicrob Agents Chemother. 2015;59(10):6581-7.

35. Choi YJ, Kim Y, Yong D, Byun J, Kim TS, Chang YS. Candida parapsilosis. Emerg Infect Dis. 2018;24(9): 1768-70.

36. Cappelletty D, Eiselstein-McKitrick K. The echinocandins. Pharmacotherapy. 2007;27:369-88.

37. Hinske LC, Weis F, Heyn J, Hinske P, Beiras-Fernandez A. The role of micafungin and anidulafungin in the treatment of systemic fungal infections: applications and patents for two novel echinocandins. Recent Pat Antiinfect Drug Discov. 2012;7:1-7. 
38. Luque S, Campilo N, Álvarez-Lerma F, Ferrández O, Horcajada JP, Grau S. Pharmacokinetics of micafungin in patients with pre-existing liver dysfunction: a safe option for treating invasive fungal infections. Enferm Infect Microbiol Clin. 2016;34(10):652-4.

39. https://www.accessdata.fda.gov/drugsatfda_docs/label/ 2019/021506s023lbl.pdf. Accessed on 1 Jan 2020.

40. Vekeman F, Weiss L, Aram J, et al. Retrospective cohort study comparing the risk of severe hepatotoxicity in hospitalized patients treated with echinocandins for invasive candidiasis in the presence of confounding by indication. BMC Infect Dis. 2018;18(1):438.

41. Thomson SJ, Cowan ML, Johnston I, Musa S, Grounds M, Rahman TM. 'Liver function tests' on the intensive care unit: a prospective, observational study. Intensive Care Med. 2009;35:1406-11.

42. Huang HY, Lu PL, Wang YL, Chen TC, Chang K, Lin SY. Usefulness of EQUAL Candida scores for predicting outcomes in patients with candidemia: a retrospective cohort study. Clin Microbiol Infect. 2020. https://doi.org/10.1016/ j.cmi.2020.01.029.

43. Arendrup MC, Perlin DS. Echinocandin resistance: an emerging clinical problem? Curr Opin Infect Dis. 2014;27:484-92.

Publisher's Note Springer Nature remains neutral with regard to jurisdictional claims in published maps and institutional affiliations. 